LA-UR-01-4760

Approved for public release; distribution is unlimited.

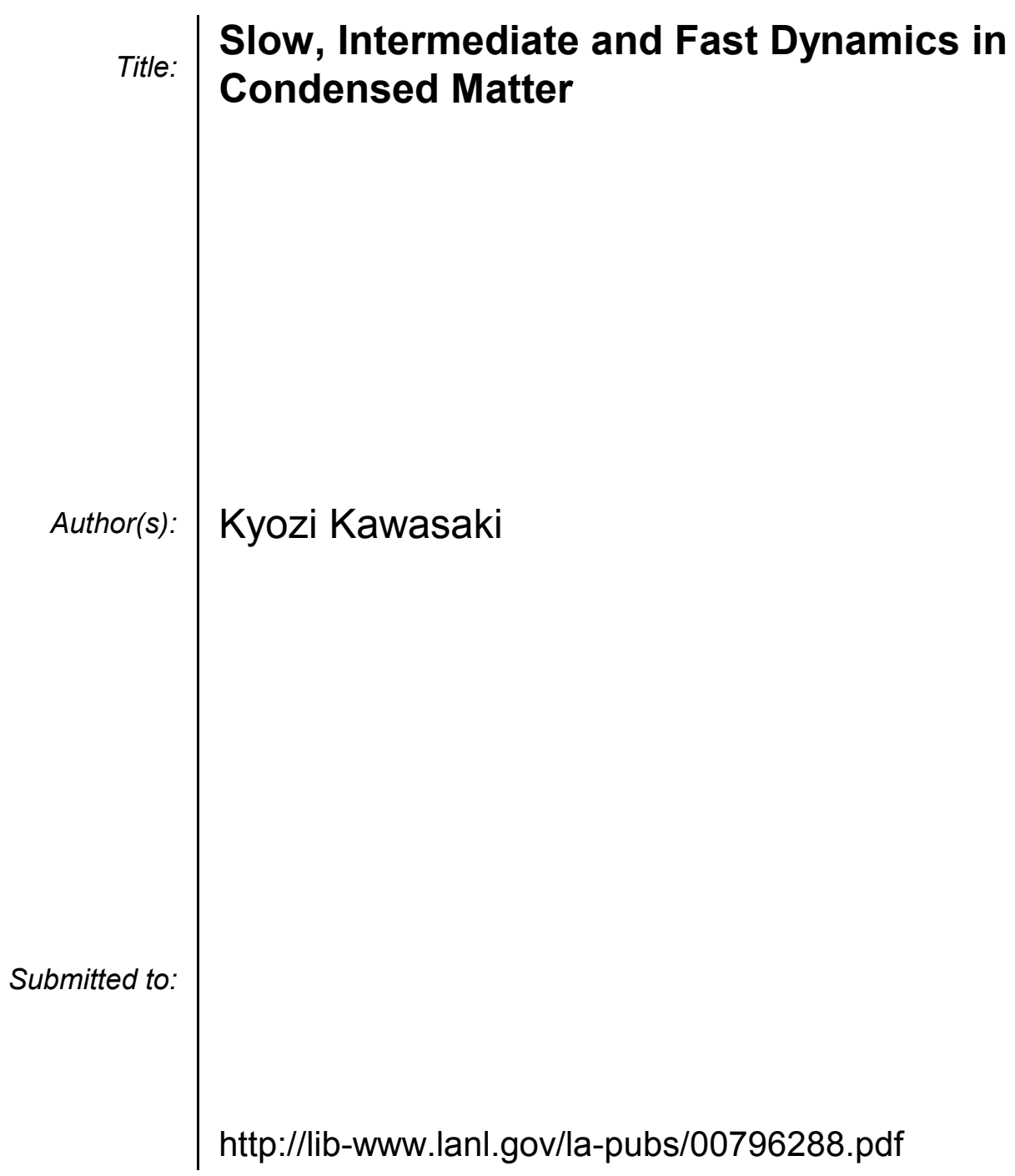

Los Alamos National Laboratory, an affirmative action/equal opportunity employer, is operated by the University of California for the U.S. Department of Energy under contract W-7405-ENG-36. By acceptance of this article, the publisher recognizes that the U.S. Government retains a nonexclusive, royaltyfree license to publish or reproduce the published form of this contribution, or to allow others to do so, for U.S. Government purposes. Los Alamos National Laboratory requests that the publisher identify this article as work performed under the auspices of the U.S. Department of Energy. Los Alamos National Laboratory strongly supports academic freedom and a researcher's right to publish; as an institution, however, the Laboratory does not endorse the viewpoint of a publication or guarantee its technical correctness. 


\title{
Slow, Intermediate and Fast Dynamics in Condensed Matter
}

\author{
Kyozi Kawasaki* \\ CNLS, Los Alamos Nantional Laboratory \\ Los Alamos, NM 87545, USA
}

\begin{abstract}
:
As is well-known, in gas dynamics there are three levels of descriptions: microscopic, mesoscopic and macroscopic. The corresponding equations are: Liouville, Boltzmann, and hydrodynamic equations, respectively. The difficulty of condensed matter such as classical liquids is general absence of well-defined mesoscopic level of descrition which is a scaffold so to speak enabling us to jump from microscopic to macroscopic descritions. However, there are certain circumstances in which this is possible as we illustrate for critical dynamics and phase ordering kinetics.
\end{abstract}

\section{Introduction}

It is an unexpected great honor for me to be awarded a Boltzmann medal of the year 2001. Colleagues who congratulated me often remarked that the award is overdue which I do not know is true, even though the remark certainly applies to Bernie Alder. However, this remark does imply that what I am going to write is a rather old story essentially spanning from the early sixties to to the early eighties.

Statstical physics initiated by Maxwell, Boltzmann, and Gibbs more than a hundred years ago was originally aimed at understanding macroscopic behavior of matter in terms of constituent atoms and molecules. In other words, one tries to explain macroscopic slow dynamics in terms of microscopic fast dynamics. In non-equilibrium domain, a culmination of this efforts resulted in establisment of general linear response theory during the period from the mid fifties to the early sixties. It was believed that this framework gives a derivation of macroscopic laws like hydrodynamics at least in the linear regime together with the exact microscopic expressions for transport coefficients, known under the name of Green-Kubo formulae[1]. This formula for an Onsager kinetic coefficient $L$ typically takes the form,

$$
L=\int_{0}^{\infty}<J(t) J(0)>d t
$$

Here $L$ enters macroscopic laws with macroscopic time scales whereas $J(t)$ describes microscopic processes with microscopic time scales. The linear response theory which led to the Green-Kubo formulae implicitly assumes that macroscopic and microscopic time scales are well separated and there is nothing in between. This assumption guarantees, for instance, convergence of the GreenKubo integral expression giving rise to finite transport coefficients at all times.

Indications that the situation was not so simple started to appear already from the mid sixties ${ }^{1}$. This can be imagined also from the fact that analytical computation of transport coefficients for realistic systems like classical liquids based on the Green-Kubo formulae are impossible in most cases, which undermines the above-mentioned derivation of macroscopic laws. It is now understood that the assumption of clear-cut separation of time scales of macroscopic and microscopic degrees

\footnotetext{
*Permanent address: 4-37-9 Takamidai, Higashi-ku, Fukuoka 811-0215, Japan

${ }^{1}$ In fact one of the earliest indications was discovedred in the molecular dynamics work of Bernie Alder. Status of the Green-Kubo formulae and its problems were discussed in [2].
} 
of freedom, which is the basis of the above-mentioned derivation of macroscopic laws, is no longer true. We now know that the the degrees of freedom whose time scales are neither microscopic nor macroscopic constitute an important independent entity. This class of the degrees of freedom may be called the mesoscopic degrees of freedom.

The oldest and clearest example where mesoscopic degrees of freedom play a major role is gas dynamics. Here the mean free time has a mesoscopic character and the appropriate equation corresponding to this time scale is just the Boltzmann equation. Even for this simple case some unexpected subtleties were discovered in the mid sixties in an attempt to obtain density corrections of transport coefficients. This topic will be summarized in the next section.

The next example is critical dynamics. Here the mesoscopic degrees of freedom are associated with critical fluctuations which can extend to thousands of Angstroms. Therefore, one can expect to develop detailed analytical treatment of critical dynamics which is comparable to what was achieved for gas dynamics. This will be described in the third section.

The last example is the late stage dynamics of phase ordering. Here the mesoscopic degrees of freedom are represented by pattterns formed by topological defects like domain walls and vortex lines.

\section{Gas dynamics}

Nonequilibrium statistical physics pioneered by Maxwell and Boltzmann resulted in the Boltzmann equation derived by heuristic considerations more than a hundred years ago . The first principle derivation of this equation came only at the end of the second world war in 1945-46 in the hand of Bogoliubov².

One starts from the $N$-body phase space distribution function $\hat{f}_{N}$ which obeys the Liouville euqation. From this one can form $s$ body reduced distribution functions with $s=1,2, \cdots$. The latter obey the exact BBGKY hierarchy which are, for the case of the two-body intermolecular forces:

$$
\begin{array}{r}
\frac{\partial}{\partial t} f_{1}=\Phi_{1}\left(f_{1}, f_{2}\right) \\
\frac{\partial}{\partial t} f_{2}=\Phi_{1}\left(f_{1}, f_{2}, f_{3}\right) \\
\ldots
\end{array}
$$

This hierachy has to be closed to find solution. The crucial observation of Bogolyubov is that for a dilute gas with $n^{*} \equiv(N / V) \times($ molecular volume $) \ll 1, f_{1}(t)$ is slowly varying as compared to the other $f$ 's where $V$ is the system volume. This observation led Bogoliubov to assume that $f_{j}$ with $j>1$ depends on time only through $f_{1}$ (the Bogolyubov fusnctional ansatz). Substitution of the above ansatz into the BBGKY hierarchy yields closed equations for $f_{1}$ which is expanded in powers of $n^{*}$. One expects to obtain the higher order density correction to the Boltzmann equation by simply carrying out this procedure to higher orders in $n^{*}$. The transport coefficients are then obtained by successive expansions of the Boltzmann equation and its extensions that include the density corrections about a local equilibrium $f_{1}$ in terms of gradients (Chapman-Enskog procedure). Since $n^{*} \propto n=$ (density) this way we should get the density expansion (or the virial expansion) for transport coefficients, e.g. for shear viscosity $\eta(n)$ :

$$
\eta(n)=\eta_{0}+\eta_{1} n+\eta_{2} n^{2}+\cdots
$$

When people tried to actually evaluate the coefficients like $\eta_{1}$ and $\eta_{2}$, unexpected surprise occurred. For instance, the integral that enters the computation of $\eta_{1}$ of two-dimensional gas diverges logarithmically with the system size. For three-dimensional gas such divergence first occurs for $\eta_{2}$. Such divergences are due to the fact that the events where double and triple

\footnotetext{
${ }^{2}$ The material in this section was reviewed with proper references, for instance, in [3].
} 
collisions with particles separated by distances much longer than mean free path dominate in these integrals, respectively. Then cure to such difficulties is obvious: indefinite numbers of other particles intervene in the collision paths to convert logarithmic divergence into logarithm of the mean free path. Such complicated processes form ring collisions.

The end results are

For two-dimension:

$$
\eta(n)=\eta_{0}+\eta_{1} n \ln \left(1 / n \sigma^{2}\right)+\cdots
$$

For three-dimension:

$$
\eta(n)=\eta_{0}+\eta_{1} n+\eta_{2} n^{2} \ln \left(1 / n \sigma^{3}\right)+\cdots
$$

The above result demonstrates that in non-equilibrium situation, in contrast to equilibrium situation, even gas dynamics cannot be closed with processes involving finite numbers of molecules, and indefinite numbers of molecules are inevitably involved. This prompts us to shift our attention to the other extreme: critical phenomena.

\section{Critical dynamics}

Crtical phenomena occur as a result of delicate balance of repulsive and attractive interactions among large numbers of molecules. The most spectacular effect observed in systems near criticality is appearence of spatially extended crtical fluctuations whose length scale can easily reach thousands of $\AA$. This is associated with the fact that a system becomes infinitely "soft" as critcality is approached. For a liquid-gas phase transition, the isothermal compressibility diverges with some exponent $\gamma$ as $\left|T-T_{c}\right|^{-\gamma}$. Intensity of critcal fluctuations are directly measured by scattering experiments which yield scattering structure factor of the form

$$
S(k) \equiv<\rho_{\mathbf{k}} \rho_{-\mathbf{k}}>\propto \frac{1}{k^{2}+\xi^{-2}}
$$

where $\rho_{\mathbf{k}}$ is the Fourier component of the density fluctuation, $\xi$ is the correlation length of critcal fluctuations behaving as $\left|T-T_{c}\right|^{-\nu}$, and $\left\langle\cdots>\right.$ stands for equilibrium average ${ }^{3}$.

\subsection{Early theoretical attempts}

The main issue of critical dynamics is to understand effects of these static crtical anomalies on dynamical properties like transport coefficients. The oldest proposal put forward was to attribute whatever the critcal anomalies entirely to static quantities that appear in the theory. As a simple example let $s$ be an order parameter like magnetization subtracted by its equilibrium average value ( which may depend on a wave vector ). The relaxation equation for $s$ may take the form, denoting time derivative by an upper dot,

$$
\dot{s}=-\Gamma s
$$

Since the rhs should be proportional to the thermodynamics driving force acting on $s$, that is, to $\chi^{-1} s$ where $\chi$ is the susceptibility in a generalized sense, we can write for the relaxation rate $\Gamma$

$$
\Gamma=L \chi^{-1}
$$

where $L$ is the Onsager kinetic coefficient. The simple-minded theory of crtical dynamics, which is known under the name of the conventional theory, assumes that $L$ has no critcal anomaly and hence a finite quantity[4]. As a consequence the theory predicts

$$
\Gamma \propto\left|T-T_{c}\right|^{\gamma}
$$

This theory which dates from the mid fifties was used to analyze the results of the very first inelastic magnetic neutron scattering experiments near criticality of 1960[5]. In those days this

\footnotetext{
${ }^{3}$ This is the approximate Ornstein-Zernike form. In the same approximation, we have $\gamma=2 \nu$. Here we will not be concerned with subtle corrections to this.
} 
simple theory appeard to be quite sufficient since the graph plotting the measrued spin diffusion coefficient as a function of temperature had three data points with huge error bars. Therefore it was easy to draw a straight line passing through these error bars and crossing the horizontal axis at $T_{c}$.

\subsection{Mode coupling theory (MCT)}

Let us now ask the question: can Onsager coefficients always remain finite near criticality? In the days when no theory was available to answer this question, we had to first look at the experimental situation. If we turn our eyes from magnets to fluids, typical Onsager kinetic coefficients are the thermal condauctivity $\lambda$ and the shear viscosity $\eta^{4}$. The experiments have clearly shown that the thermal conductivity of fluids, in particular, carbon dioxyde, increses sharply as criticality is approached, whereas shear viscosity shows only a mild hump near criticality. Therefore, a theory is needed to account for critical anomalies of Onsager kinetic coefficients themselves.

The first suggestion of how one could deal with this problem came from Marshall Fixman in a paper published in 1962[7]. In that paper he showed that the shear viscosity can be calculated if we know static correlation of the order parameter fluctuations (3.1) and the diffusion law for the order parameter. This idea was incorporated in the framework of the linear response theory of section 1 by the author[8]. Subsequently Kadanoff and Swift cast the theory in a more usable form [9]. This is the beginning of the present mode coupling theory.

In the following we give a simple accout of this theory for thermal conductivity $\lambda$ of onecomponent liquid. Our starting point is the following Green-Kubo formula for $\lambda$ :

$$
\lambda=\frac{1}{k_{B} T^{2} V} \int_{0}^{\infty} d t \int d \mathbf{r} \int d \mathbf{r}^{\prime}<j_{x}(\mathbf{r}, t) j_{x}\left(\mathbf{r}^{\prime}, 0\right)>
$$

where $V$ is the system volume, $j_{x}(\mathbf{r}, t)$ is the $\mathrm{x}$-component of microscopic expression for the heat flux density at the location $\mathbf{r}$ and the time $t$. This expression is exact whenever a finite thermal conductivity exists. Even though each term in the expression of $j_{x}(\mathbf{r}, t)$ contains a finite number of molecules ( 2 for the case of two-body inter molecular forces) at $t=0$, the time dependence brings in variables referring to all other molecules. This makes direct computaion of expressions like (3.5) practically impossible. In this respect, vicinity of criticality does not help but enhances the difficulty because the large correlation length $\xi$ tends to bring in more molecules to interact. The idea of mode coupling approach is to give up direct computation of this complicated quantity but to make use of the fact that near criticality fluctuations having large length scale comparable to $\xi$ dominate the scene. That is, to split $j_{x}(\mathbf{r}, t)$ into the two parts as

$$
j_{x}(\mathbf{r}, t)=\left[j_{x}(\mathbf{r}, t)\right]_{0}+\left[j_{x}(\mathbf{r}, t)\right]_{c}
$$

where $\left[j_{x}(\mathbf{r}, t)\right]_{c}$ represents the part containing fluctuations whose wave lengths are of the order of $\xi$ and the remainder is included in $\left[j_{x}(\mathbf{r}, t)\right]_{0}$. Here one assumes that this separation can be done in a clean way so that $\left[j_{x}(\mathbf{r}, t)\right]_{0}$ no longer contains critical fluctuations. We further assumews that cross terms of the two when (3.6) is substituted into (3.5) can be dropped. This is correct if long wavelngth fluctuations and short wavelength fluctuations do not interfere each other.

Now, if we know that $\lambda$ tends to diverge at criticality as suggested by experimental findings, such a divergence can come only from $\left[j_{x}(\mathbf{r}, t)\right]_{c}$. So our next question is how to find a proper expression for $\left[j_{x}(\mathbf{r}, t)\right]_{c}$. A standard formal way is to use a projection operator to project this quantity onto products of the gross variables, i.e. slowly varying variables consisting of the densities of conserved quantities and, if necessary, the local order parameters. Here it is important to note that no terms linear in gross variables occur in the projection due to the nature of flux $J(t)$ in the Green-Kubo formula (1.1). However, here we take a more intuitive approach. So, imagine a fluid near criticality with large scale density fluctuations $\delta \rho(\mathbf{r}, t)$. Since we are interested in thermal conductivity, it is more useful to consider local specific entropy fluctuation $\delta s(\mathbf{r}, t)$ which also plays

\footnotetext{
${ }^{4}$ Measuements of tranpost anomalies in fluids are reviewed in [6].
} 
the role of the order parameter fluctuation ${ }^{5}$. The heat content per unit volume is then $\rho_{0} T \delta s(\mathbf{r}, t)$ after subtracting a uniform thermal average where $\rho_{0}$ is the average density. This heat content is carried by fluctuating velocity field $\mathbf{v}(\mathbf{r}, t)$. These considerations lead to the following expression for the heat flux density:

$$
\left[j_{x}(\mathbf{r}, t)\right]_{c}=\rho_{0} T \delta s(\mathbf{r}, t) v_{x}(\mathbf{r}, t)
$$

Substituting this expression into (3.5) where the noncritical part containing $\left[j_{x}(\mathbf{r}, t)\right]_{0}$ is dropped, we find for the critical part $\lambda_{c}$ of the thermal conductivity,

$$
\lambda_{c}=\frac{\rho_{0}^{2}}{k_{B}} \int_{0}^{\infty} d t \int d \mathbf{r}<\delta s(\mathbf{r}, t) v_{x}(\mathbf{r}, t) \delta s(\mathbf{0}, 0) v_{x}(\mathbf{0}, 0)>
$$

A further simplifying assumtion is statistical independence of specific entropy fluctuation and velocity field. We then find

$$
\lambda_{c}=\frac{\rho_{0}^{2}}{k_{B}} \int_{0}^{\infty} d t \int d \mathbf{r}<\delta s(\mathbf{r}, t) \delta s(\mathbf{0}, 0)><v_{x}(\mathbf{r}, t) v_{x}(\mathbf{0}, 0)>
$$

Next we make use of the fact that (1) $\delta s(\mathbf{r}, t)$, being the order parameter, is varying very slowly compared to the velocity field and can be replaced by $\delta s(\mathbf{r}, 0)$, and $(2)<v_{x}(\mathbf{r}, t) v_{x}(\mathbf{0}, 0)>$ can be obtained by solving the Navier-Stokes equation ${ }^{6}$ since only its part varying smoothly in space enters due to the presence of a factor $\langle\delta s(\mathbf{r}) \delta s(\mathbf{0})>$. In this way we find

$$
\begin{aligned}
<\delta s(\mathbf{r}) \delta s(\mathbf{0})> & \propto \frac{e^{-r / \xi}}{r} \sim \frac{1}{\xi} \\
<v_{x}(\mathbf{r}, t) v_{x}(\mathbf{0}, 0)> & \propto \frac{e^{-\left[r^{2} /\left(\frac{t \eta}{\rho}\right)\right]}}{t^{3 / 2}} \sim \frac{1}{\xi^{3}}
\end{aligned}
$$

where we have also indicated power counting after $\sim$ sign since here we only want to show the power law of the divergence of $\lambda$. For the same purpose we can also make the replacements as $\int d \mathbf{r} \sim \xi^{3}, \int d t \sim t \sim \xi^{2}$ in the expression (3.9). All and all we end up with

$$
\lambda_{c} \sim \xi
$$

This is the central result of this subsection where the name mode coupling comes from the fact that (3.7) shows splitting of heat current (lhs) into product of specific entropy fluctuation and velocity field (rhs).

The total thermal conductivity $\lambda$ is the sum of the critical part which we have dealt with and the noncritical part $\lambda_{0}$ cominfg from $\left[j_{x}(\mathbf{r}, t)\right]_{0}$ :

$$
\lambda=\lambda_{0}+\lambda_{c}
$$

Often $\lambda_{0}$ is called the bare thermal conductivity and $\lambda$ the renormalized thermal conductivity since the latter is what is observed experimentally.

Physically, enhancement of heat transport arises from a large "droplet" of high heat content of the zise $\xi$ being carried by fluctuating velocity field. This then implies the novel effect to be described now. The usual Fourier law of heat transport says that heat current density at a point $\mathbf{r}$ is proportional to the temperature gradient at that point $\boldsymbol{\nabla} T(\mathbf{r})$. Now, if a heat current is carried by a large "droplet" of the size $\xi$, it is possible that heat currrent density at a point $\mathbf{r}$ can depend on the temperature gradient at another point $\mathbf{r}^{\prime}$ which is located about a distance $\xi$ away from $\mathbf{r}$. In other words, the usual Fourier's law $\overline{\mathbf{j}}(\mathbf{r})=-\lambda \nabla T(\mathbf{r})$, an upper bar denoting a nonequilibrium average, must be generalized to the following nonlocal form:

$$
\overline{\mathbf{j}}(\mathbf{r})=-\int d \mathbf{r}^{\prime} \Lambda\left(\mathbf{r}-\mathbf{r}^{\prime}\right) \nabla^{\prime} T\left(\mathbf{r}^{\prime}\right)
$$

\footnotetext{
${ }^{5}$ The density fluctuation can be written as a linear combination of two terms proportional to the specific entropy fluctuation and to the pressure fluctuation. The latter propagates away with the sound velocity and does not contribute to the slow heat conduction.

${ }^{6}$ Note that we can assume the fluid to be incompressible: $\boldsymbol{\nabla} \cdot \mathbf{v}=0$ because the longitudinal component of $\mathbf{v}$ will propagate away as a sound wave, which we have excluded.
} 
where $\Lambda(\mathbf{r})$ is a function expressing nonlocality of heat transport extending to distances of the order of $\xi$. This gives an example showing that critical anomlies are not limited to singular behavior of transport coefficients as functions of thermodynamic state variables. Other examples include singular behavior as a function of thermodynamics driving force like temperature gradient or externally applied shear rate. In the next subsection, we outline an extension of MCT to deal with such novel situations.

\section{Mesoscopic kinetic equation}

The mode coupling theory which was described in the preceding section incorporated mesoscopic degrees of freedom associated with critcal fluctuations into calculation of transport coeffiecients via the Green-Kubo formulae. As indicated in that section, critcal anomalies are not limited to transport coefficents, which necessitates development of general kinetic theory that can specifically deal with mesoscopic degrees of freedom. This equation should play the same role as the Boltzmann equation in gas dynamics. The Boltzmann equation has been used not just for computing transport coefficents of gas, but can deal with situations where no hydrodynamic equation exists such as shock waves.

Here we develop such a kinetic equation appropriate to critical fluid dynamics. Some aspects of critcal dynamics can be compared with that of fully-developed Navier-Stokes turbulence where one tries to predict statistical properties of the velocity field of a fluid on the basis of the nonlinear Navier-Stokes equation[10]. Both problems have to deal with dissipative dynamics of nonlinear fields. A vesrion of such theoretical attempt was put forward by Wyld in a form more familiar to statisatical physicist[11] where the starting point is the Navier-Stokes equation supplemented with a thermal noise. An analogous starting point for critical dynamics can be set up by taking nonlinear continuum equation satisfied by gross variables supplemented with thermal noise terms. The latter is coupled to the bare transport coefficients in the equation through the fluctuationdissipation relathions. Although general formal framework to derive such equations exists[12], here we prefer to adopt a simple approach[13]. So we write down the nonlnear Langevin equation for gross variables (also called mesoscopic kinetic equation in this context) for single-component critical fluid where gross variables are taken to be Fourier components of local specific entropy fluctuation and local velocity field $s_{\mathbf{k}}$ and $\mathbf{v}_{\mathbf{k}}$, respectively:

$$
\begin{gathered}
\left(\frac{\partial}{\partial t}+k^{2} \frac{\lambda_{0}}{\rho_{o} c_{p}(k)}\right) s_{\mathbf{k}}(t)=-i \mathbf{k} \cdot \int_{\mathbf{q}} s_{\mathbf{q}}(t) \mathbf{v}_{\mathbf{k}-\mathbf{q}}(t)+R_{s \mathbf{k}} \\
\left(\frac{\partial}{\partial t}+k^{2} \frac{\eta_{0}}{\rho_{o} c_{p}(k)}\right) \mathbf{v}_{\mathbf{k}}(t)=-\frac{T}{2} \int_{\mathbf{q}}\left[c_{p}^{-1}(\mathbf{q})-c_{p}^{-1}(\mathbf{k}-\mathbf{q})\right] i\left[\mathbf{q}-\frac{\mathbf{k}}{k^{2}}(\mathbf{q} \cdot \mathbf{k})\right] s_{\mathbf{k}-\mathbf{q}}(t) s_{\mathbf{q}}(t)+R_{v \mathbf{k}}(t)
\end{gathered}
$$

where $c_{p}(k)$ is the wavenumber dependent specific heat proportional to $\left\langle\left|s_{\mathbf{k}}\right|^{2}>\right.$ and the $R$ 's are the thermal noises satisfying the following fluctuation-dissipation relationships:

$$
\begin{aligned}
<R_{s \mathbf{k}}(t) R_{s-\mathbf{k}}\left(t^{\prime}\right)> & =2 k_{B} T \lambda_{0} k^{2} \delta\left(t-t^{\prime}\right) \\
<\mathbf{R}_{v \mathbf{k}}(t) \mathbf{R}_{v-\mathbf{k}}\left(t^{\prime}\right)> & =2 k_{B} T \eta_{0} k^{2}(\mathbf{1}-\hat{\mathbf{k}} \hat{\mathbf{k}}) \delta\left(t-t^{\prime}\right)
\end{aligned}
$$

with 1 the unit dyadic and $\hat{\mathbf{k}} \equiv \mathbf{k} /|\mathbf{k}|$. This sort of kinetic equations are starting points for computing quantities of physical interests such as order parameter time correlation functions. We illustrate this for the case of relaxation rate $\Gamma_{k}$ of $\bar{s}_{\mathbf{k}}$ which can be also obtained from the timecorrelation function. The result is

$$
\Gamma_{k}=\frac{k_{B} T}{6 \pi \eta \xi^{3}} F(k \xi)
$$

with the scaling function $F(x)$ given by

$$
F(x) \equiv \frac{3}{4}\left[1+x^{2}+\left(x^{3}-x^{-1}\right) \tan ^{-1} x\right]
$$


This result interpolates the hydrodynamic region $k \xi \ll 1$ and the critical region $k \xi \gg 1$ where we find

$$
\Gamma_{k} \approx\left\{\begin{array}{cc}
D k^{2} & \text { for } \quad k \xi \ll 1 \\
\frac{k_{B} T}{16 \eta} k^{3} & \text { for } \quad k \xi \gg 1
\end{array}\right.
$$

with

$$
D \equiv \frac{k_{B} T}{6 \pi \eta \xi}
$$

This theoretical prediction[14] was first tested by the Bergé group[15], which subsequently led to further refinements of theory and experiments.

The result (4.7) was interpreted in terms of the Einstein-Stokes law of diffusion coefficient of hard spheres immersed in a fluid with shear viscosity $\eta$ where no slip boundary condition was assumed for the surface of hard spheres[16]. Although this interpretation is qualitatively correct, the agreement of the numerical coefficient must be considered as fortuitous.

\section{Recent developments of critical fluid dynamics}

After mode coupling theory it is natural to refine the theory using the powerful renormlization group (RG) ideas. This has been done and is reviewed in [17]. The most delicate problem was to understand a weak critical divergence of shear viscosity. MCT predicts a logarithmic divergence. However, more accurate RG calculation that expands around 4 dimension with the expansion parameter $\epsilon \equiv 4-d$ showed a weak power law divergence with the critical exponent $\epsilon \nu / 19$. Refined RG calculation of frequency and wavenumber dependent shear viscosity has been favorably compared with the recent high precision measurements under microgravity environments[18]. This result demonstrates beyond any doubt the essential soundness of the ideas leading to MCT.

\section{H-theorem}

It is of some interest to consider an analogue of the Boltzmann H-theorem for the mesoscopic kinetic equation. For this purpose we write down this equation for the general gross variables $\{a\} \equiv a_{1}, a_{2}, \cdots$ in the following Fokker-Planck form for the probability distribution function $D(\{a\}, t)$ :

$$
\frac{\partial}{\partial t} D(\{a\}, t)=-\sum_{i} \frac{\partial}{\partial a_{i}} v_{i}(\{a\}) D(\{a\}, t)+\sum_{i j} \frac{\partial}{\partial a_{i}} L_{i j}^{0} k_{B} T D_{e}(\{a\}) \frac{\partial}{\partial a_{j}} D(\{a\}, t)
$$

where $v_{i}(\{a\})$ is the instantaneous ( reversible) rate of change of $a_{i}, L_{i j}^{0}$ the bare Onsager kinetic coefficient and $D_{e}(\{a\}) \propto \exp \left[-H(\{a\}) / k_{B} T\right]$ the equilibrium probability distribution function with $H(\{a\})$ the free energy function.

Since the system is in contact with a constant temperature heat bath, the quantity that is not expected to decrease in time is not the system entropy $S(t)$ but the Mathieu function $M(t) \equiv$ $S(t)-U(t) / T$ with $U(t)$ the "internal energy", that is, the nonequilibrium average of $H(a)$ and $S(t)$ the entropy as usually defined with respect to $D(\{a\}, t)$. Then we find the following after some algebra:

$$
\frac{d}{d t} M(t)=k_{B} \int d\{a\} \frac{D_{e}(\{a\})^{2}}{D(\{a\}, t)} \sum_{i j} k_{B} T L_{i j}^{0}(\{a\})\left(\frac{\partial}{\partial a_{i}} \frac{D(\{a\}, t)}{D_{e}(\{a\})}\right)\left(\frac{\partial}{\partial a_{j}} \frac{D(\{a\}, t)}{D_{e}(\{a\})}\right)
$$

This quantity is non-negative since the matrix formd by $L_{i j}^{0}$ is non-negative. Hence the H-theorem is $^{7}$.

$$
\frac{d}{d t} M(t) \geq 0
$$

It is noteworthy that only the bare Onsager kinetic coefficients appear in the above expression which does depend on the degree of coarse graining used to define the gross variables in the first place.

\footnotetext{
${ }^{7}$ The H-theorem for systems in contact with heat bath of the kind used here is known for general master equation with detailed balance[19].
} 


\section{Critical fluid under shear flow}

The mesoscopic kinetic equation in the preceding section can deal with problems far away from equilibrium. As an example we treat the critical fluid under steady shear flow.

If a steady flow $u_{x}(y)$ is directed along the x-axis, the rate of shear is $\mathcal{D} \equiv d u_{x}(y) / d y$. If we denote by $\Gamma_{k}$ be the natural decay rate of critical fluctuation with wave number $\mathrm{k}$ and is given by (4.6), $\Gamma_{k} \approx k_{B} T_{c} /\left(16 \eta k^{3}\right)$ for $k \xi \gg 1$ there is a critical wave number $k_{c} \equiv\left(16 \eta D / k_{B} T_{c}\right)^{1 / 3}$ such that those critical fluctuations with $k<k_{c}$ are strongly affected by shear.

This problem was taken up alsmost simultaneously around 1979 by the experimetal group of Daniel Beysens[20] and theoretically by Onuki and myself[21] . The experiments on a binary fluid (cyclohexane-anilline) have shown that

1. Lowering of critical temperature with shear rate:

$$
T_{c}(\mathcal{D})-T_{c}(0)=-1.8 \times 10^{-4}|\mathcal{D}|^{0.53}, \quad\left(\mathcal{D} \text { in } \mathrm{cm}^{-1}\right)
$$

2. Anisotropy in the measured wavenumber dependent susceptibility $\chi_{\mathbf{k}}$ which is more pronounced as $|\mathcal{D}|$ increases.

3. Reduction of turbidity with increasing $|\mathcal{D}|$

The relevant mesoscopic kinetic equation to study this problem is obtained by supplementing the rhs of (4.1) with:

$$
\cdots-D y \frac{\partial s(\mathbf{r}, t)}{\partial x}
$$

We here list some consequences of this theory.

- Naïve mean field calculation already gives the anisotropic wavenumber dependent susceptibility

$$
\chi_{\mathbf{k}}=\left(\xi^{-2}+k_{c}^{8 / 5}\left|k_{x}\right|^{2 / 5}+k^{2}\right)^{-1}
$$

One can see that critical fluctuations with $k_{x} \neq 0$ are suppressed.

- Renormalization group calculation for small $\epsilon=4-d$ can be carried out and we found the scaling form for $\chi_{\mathbf{k}}$ as follows

$$
\chi_{\mathbf{k}}=\xi_{\perp}^{2} \mathcal{F}\left(k_{x} \xi_{\|}, k_{y} \xi_{\perp}, k_{z} \xi_{\perp}\right)
$$

where $\mathcal{F}$ is a complicated scaling function depending on three variables reflecting the anisotropy and

$$
\begin{aligned}
\xi_{\perp} & \equiv \xi_{0}\left(k_{c} \xi_{0}\right)^{(1-\gamma) / 2 \nu}\left\{\left[T-T_{c}(\mathcal{D})\right] / T_{c}(0)\right\}^{-1 / 2} \\
\xi_{\|} & \equiv \xi_{\perp} \cdot\left(k_{c} \xi_{\perp}\right)^{4}
\end{aligned}
$$

where the shear-dependent critical temperature is given by

$$
T_{c}(\mathcal{D})=T_{c}(0)-1.3 \times 10^{-4}|\mathcal{D}|^{1 / 3 \nu}
$$

A measure of anisotropy is then given by

$$
\xi_{\|} / \xi_{\perp}=\left(k_{c} \xi_{\perp}\right)^{4}=\left(k_{c} \xi_{0}\right)^{2 / \nu}\left\{\left[T-T_{c}(\mathcal{D})\right] / T_{c}(0)\right\}^{-2} \gg 1
$$

The anisotropic contour of the equal light scattering intensity was named "Moustache effect" by Beysens. 


\section{Phase ordering kinetics}

The major difference in the problems of phase ordering $(\mathrm{PO})$ and critical dynamics $(\mathrm{CD})$ is the following ${ }^{8}$ :

- Amplitudes of the local order parameter fluctuations in PO are large, close to the saturation values in the two phase coexistence, whereas in $\mathrm{CD}$ such amplitudes are very small due to proximity of second order phase transition. Only spatial extents of a fluctuationg region tend to diverge in the latter.

- PO is generally characterized by two length scales. One is the domain wall thickness which ranges from micorscopic length at low temperatures to thousands of Ångstroms near criticality. The other length is that characerizes geometry of domain patterns. This includes the average radius of curvature of domain walls and distances between neighboring domain walls. In CD only the correlation length of critcal fluctuations come in excluding the obvious microscopic length.

Therefore, theoretical approaches to these two problems are quite different. In particular, renormalization group method which was so powerful for critical phenomena appears to be not so much useful in PO.

If one is interested in the so-called late stage of phase ordering kinetics where local phase separation is basically completed, what remains is the slow change of domain patterns. Hence the starting point of the late stage dynamics is the equation of motion of domain walls. Here the "microscopic regime" can be either genuine microscopic equation of motion or dissipative field equation like TDGL or Cahn-Hilliard equation. The mesoscopic kinetic equation is the one describing dynamics of topological defects like domain walls. Then, what corresponds to macroscopic regime is described by time-dependent scattering structure factors or some macroscopic properties of phase separating systems. Now, the simplest example of such mesoscopic kinetic equation in this case when the order parameter is not conserved, such as that associated with the order-disorder transition of binary alloys, is the Allen-Cahn equation of domain wall velocity of the form

$$
v=L^{\prime} K
$$

where $v$ is the interface velocity, $L^{\prime}$ a kinetic coefficients, and $K$ the interfacial mean curvature. Even such a simple-looking equation is rather difficult to solve.

A useful approach is to rewrite this equation by introducing a level field $u(\mathbf{r}, t)$ by demanding $u(\mathbf{r}, t)=0$ for $\mathbf{r}$ right on the interface at the time $t$.

This implies that $\left(\partial_{t}+\mathbf{v} \cdot \boldsymbol{\nabla}\right) u(\mathbf{r}, t)=0$ where $\mathbf{v} \equiv v \mathbf{n}$ with $\mathbf{n}=\boldsymbol{\nabla} u /|\boldsymbol{\nabla} u|$ the unit vector normal to the interface. By making use of $K=\boldsymbol{\nabla} \cdot \mathbf{n}$, the interface equation takes the following form:

$$
\frac{\partial}{\partial t} u(\mathbf{r}, t)=L^{\prime}\left(\nabla^{2}-\mathbf{n n}: \nabla \nabla\right) u(\mathbf{r}, t)
$$

This is an exact closed equation for the level field, which is, however, untractable. Note that $\mathbf{n}$ also depends on $\mathbf{r}$ and $t$.

Now, if the domain pattern is sufficiently random, we may approximate $\mathbf{n n}$ above by its directional average $(1 / d) \mathbf{1}$ where $d$ and $\mathbf{1}$ are, respectively, the dimensionality of space and unit dyadic in d-dimensional space[22]. This linearizes the equation for $u$ as

$$
\frac{\partial}{\partial t} u(\mathbf{r}, t)=L \nabla^{2} u(\mathbf{r}, t) \quad \text { with } \quad L \equiv\left(1-\frac{1}{d}\right) L^{\prime}
$$

On the other hand the order parameter field $S(\mathbf{r}, t)$ is related to the level field by

$$
S(\mathbf{r}, t)=M \operatorname{sgn}(u(\mathbf{r}, t))
$$

\footnotetext{
${ }^{8}$ Here we consider the order parameter having Ising-like symmetry.
} 
where $M$ is the saturated value of $|S|$. This implies that for the case of assumed symmetric Gaussian distribution for $u(\mathbf{r}, t)$, we have

$$
\frac{<S\left(\mathbf{r}_{1}\right) S\left(\mathbf{r}_{2}\right)>_{t}}{<S(\mathbf{r})^{2}>_{t}}=\frac{2}{\pi} \sin ^{-1} \frac{<u\left(\mathbf{r}_{1}\right) u\left(\mathbf{r}_{2}\right)>_{t}}{<u(\mathbf{r})^{2}>_{t}}
$$

where the suffix $t$ indicates the time when correlation functions are evaluated. It is reasonable to assume that right after the quench at $t=0$ there is no correlation between two different points in space. We can then take $<u\left(\mathbf{r}_{1}\right) u\left(\mathbf{r}_{2}\right)>_{t=0} \propto \delta\left(\mathbf{r}_{1}-\mathbf{r}_{2}\right)$, and the final outcome is

$$
\frac{<S\left(\mathbf{r}_{1}\right) S\left(\mathbf{r}_{2}\right)>_{t}}{<S(\mathbf{r})^{2}>_{t}}=\frac{2}{\pi} \sin ^{-1} \exp \left[-\frac{\left(\mathbf{r}_{1}-\mathbf{r}_{2}\right)^{2}}{8 L t}\right]
$$

The spatial Fourier transform of this result is the scattering structure function at time $t$ after the quench and is favorably compared with experiments and computer simulation results (See e.g. Rikvold et al [23]. ) with discrepancies in details.

Although this so-called OJK theory seems quite successful in predicting statstical properties of the order parameter fluctuations, the approximation used is totally uncontrolled. Therefore it is legitimate to ask nature of the approximation. There are arguments that the OJK theory is exact for infinite spational dimensionality [24]

On the other hand the original OJK approximation was extended to general $n$-component vector order parameter function $\mathbf{S}(\mathbf{r}$ ) and the general $d$-dimensaional space (Toyoki, Bray, and others. See [25]) with the following results:

$$
\frac{<\mathbf{S}\left(\mathbf{r}_{1}\right) \cdot \mathbf{S}\left(\mathbf{r}_{2}\right)>_{t}}{<\mathbf{S}(\mathbf{r})^{2}>_{t}}=\frac{n}{2 \pi} e^{-r^{2} / 8 D t} B\left(\frac{n+1}{2}, \frac{1}{2}\right)^{2} F\left(\frac{1}{2}, \frac{1}{2}, \frac{n}{2}+1, e^{-r^{2} / 4 D t}\right)
$$

where $B$ is the Beta function and $F$ is the confluent hypergeometric function.

It is noteworthy that the rhs is independent of $d$. The $d$-dependence comes in only after spatial Fourier transformation to get scattering structure function. This suggests the following embedding property[26]. Now, consider a case with $n>d$ where topological defects are unstable. Embed this case onto some higher dimensional space with $d^{\prime} \geq n$ where topological defects are stable. Then phase ordering in $d$-space looks like a shadow of stable topological defects in $d^{\prime}$-space. However, validity of this embedding property is limited in view of imprecise nature of the Gaussian assumption and linearization approximation made for the level field.

\section{Concluding remarks}

As a conclusion of this paper a summary of mesoscopic equations discussed here is given in the table form below.

Table 1: Mesoscopic kinetic equations

\begin{tabular}{|cccc|}
\hline system & equation & length scale & time scale \\
\hline \hline gas & Boltzmann equation & mean free path & mean free time \\
\hline critical fluid & $\left.\begin{array}{l}\text { Nonlinear Langevin or } \\
\text { Fokker-Planck equation }\end{array}\right)$ & $\xi$ & $6 \pi \eta \xi^{3} / k_{B} T$ \\
\hline $\begin{array}{l}\text { phase } \\
\text { ordering } \\
\text { kinetics }\end{array}$ & $\left.\begin{array}{l}\text { Equation of motion for } \\
\text { topological defects }\end{array}\right)$ & $\left(\begin{array}{l}\text { length characteristic of } \\
\text { patterns of topologocal } \\
\text { defects }\end{array}\right)$ & $\left(\begin{array}{l}\text { time characteristic of pat- } \\
\text { tern changes of topologo- } \\
\text { cal defects }\end{array}\right.$ \\
\hline
\end{tabular}

Acknowledgements

This research is supported by the Department of Energy, under contract W-7405-ENG-36. An additional partial support by the Cooperative Research under the Japan-U.S. Cooperative Science Program sponsored by Japan Society of Promotion of Science is also gratefully aknowledged. 


\section{References}

[1] R. Kubo, M. Toda, and N. Hashitsume, Statistical Physica II. Nonequilibrium Statistical Mechanocs, 2nd ed., 2nd printing, (Springer-Verlag, Berlin, 1995)

[2] H.J.M. Hanley, ed., Nonlinear Fluid Behavior, (North-Holland, Amsterdam, 1983)

[3] J.R. Dorfman and H. van Beijeren, in: Statistical Mechanics B, ed. B. Berne, (Plenum Press, New York, (1977)

[4] L. van Hove, Phys. Rev.95 (1954) 249, 1374; P.G. de Gennes, Report to C.E.A. de Saclay, No. 929 (1959); H. Mori and K. Kawasaki, Progr. Theor. Phys. (Kyoto), 27 (1962) 529

[5] M. Ericsson and B. Jacrot, J. Phys. Chem. Solids 13 (1960) 235

[6] J.V. Sengers, in: Critical Phenomena, M.S. Green, ed. (Academic Press, New York, 1971)

[7] M. Fixman, J. Chem. Phys. 36 (1962) 310

[8] K. Kawasaki, Phys. Rev. 150 (1966) 291

[9] L. Kadanoff and J. Swift, Phys. Rev. 166 (1968) 8

[10] Uriel Frisch,Turbulence, ( Cambridge University Press, Cambridge, 1995)

[11] H.W. Wyld, Jr. Ann. Phys. (N.Y.) 14 (1961) 143

[12] K. Kawasaki, in: Phase Transitions and Critical Phenomena, vol 5A, C. Domb and M. S. Green (eds.), Acdemic Press, London (1976) unton,

[13] K. Kawasaki and J.D. Gunton, in: Progress of Liquid Physics, C.A. Croxton, ed., (Wiley, New York, 1978)

[14] K. Kawasaki, Ann. Phys. (N.Y.) 61 (1970) 1

[15] P. Bergé, P. Calmettes, C. Laj and B. Volochine, Phys. Rev. Lett. 23 (1969) 693; ibid 24 (1970) 1223

[16] G. Arcovito, C. Faloci, M. Roberti, and L. Mistura, Phys. Rev. Lett. 22,(1969) 1040

[17] P.C. Hohenberg and B.I. Halperin, Rev. Mod. Phys. 49 (1977) 435

[18] R.F. Berg, M.R. Moldover and G.A. Zimmerli, Phys. Rev. Lett. 82 (1999) 920

[19] N.G. van Kampen, Stochastic Processes in Physics and Chemistry,(Elsevier, Amsterdam, 1992)

[20] D. Beysens, in Scattering technique applied to supramolecular and nonequilibrium systems, eds. S-H. Chen N. Chu and R. Nossal (Plenum, 1991)

[21] K. Kawasaki and A. Onuki, ibid.

[22] T. Ohta, D. Jasnow and K. Kawasaki, Phys. Rev. Lett. 49 (1982) 1223

[23] G. Brown, P. Rikvold and M. Grant, Phys. Rev. E 58 (1998) 5501

[24] C.L Emmott, Phys. Rev. E 58 (1998) 5508; however, see G. F. Mazenko, Phys. Rev. E 58 (1998) 1543.

[25] A. Bray, Adv. Phys. 43 (1994)

[26] K. Kawasaki, Vistas Astron. 37 (1993) 57 